\title{
Verifying the markers of ovarian cancer using RNA-seq data
}

\author{
TIANFENG LIU ${ }^{1}$, NINA YU ${ }^{1}$, FENG DING $^{1}$, SURONG WANG $^{1}$, SHIHONG LI ${ }^{1}$, \\ XIAOFEI ZHANG ${ }^{1}$, XIANGXIU SUN $^{1}$, YING CHEN ${ }^{1}$ and PEISHU LIU ${ }^{2}$ \\ ${ }^{1}$ Department of Gynecology, Linyi People's Hospital, Linyi, Shandong 276000; \\ ${ }^{2}$ Department of Gynecology, Qilu Hospital of Shandong University, Jinan, Shandong 250000, P.R. China
}

Received March 13, 2014; Accepted December 12, 2014

DOI: $10.3892 / \mathrm{mmr} .2015 .3489$

\begin{abstract}
Markers associated with diagnosis, presentation and potential therapeutic targets have received widespread attention in ovarian cancer research in the past few years. However, the majority of these markers have been investigated individually, and the changes in expression and the association between them are rarely documented. Next-generation sequencing, also termed RNA-seq when the sequencing targets are cDNAs, can provide a whole blueprint of the transcriptome of a specific tissue. In the present study, RNA-seq data of human ovarian cancer samples were used to verify the expression of known markers and to identify the association between them. A total of 563 markers associated with ovarian cancer were retrieved from the database of the National Center of Biotechnology Information, and used as the target markers. The transcriptome of the ovarian tissue of four different tumors, containing tumor presentation and recurrence stages, were sequenced using the Illumina GAII platform. Approximately $85.97 \%$ markers were expressed of the total 563 markers, and the majority of them were involved in pathways associated with cancer, signaling and infection. In total, 85 markers were found to be aberrantly expressed in tumor cells from patients with ovarian cancer who had recurrences, including 33 upregulated markers at the recurrence stage. Therefore, they may have roles ovarian tumor due to their aberrant expression. Differentially expressed markers and the associations between them can be assessed by examining the RNA-seq data. These findings may provide novel information for further studies on ovarian cancer.
\end{abstract}

\section{Introduction}

Ovarian cancer is one of the most common types of carcinoma in females, usually associated with a high mortality as signs and symptoms are frequently absent (1). The exact causes

Correspondence to: Dr Peishu Liu, Department of Gynecology, Qilu Hospital of Shandong University, 107 West Culture Road, Jinan, Shandong 250000, P.R. China

E-mail: peishuliu@126.com

Key words: ovarian cancer, RNA-seq data, markers of ovarian cancer remain to be elucidated. Genetic factors have been identified as important in certain ovarian cancer patients, such as mutations in the BRCA1 and BRCA2 genes, which are also risk factors for breast cancer (2). Mutations in $B R C A l$ confer a high risk of ovarian cancer and can reduce lifespan (3). In addition, marker genes of ovarian cancer can be classified into several types: Presentation $(4,5)$, recurrence $(6)$, inheritance $(7)$, prognosis $(8,9)$ and therapy targets $(10)$. Although a large number of markers have been observed to be associated with ovarian carcinoma, the incidence of and the associations and interactions between these markers have not been extensively investigated.

In the present study, all markers associated with ovarian cancer were determined by analyzing the whole transcriptome of ovarian cancer cell lines. Tumor samples were selected at two stages (presentation and recurrence) from two patients as the focus of the present study. RNAs were extracted from tumor cell lines and sequenced with Illumina GAII. By aligning RNA-seq data to marker sequences, differentially expressed markers (DEMs) were filtered out in different stages, and the interactions between them were assessed.

\section{Materials and methods}

Data sources and processing. A total of four ovarian carcinoma cell lines at different tumor stages: PEO1 (first recurrence, 22 month post chemotherapy; Patient ID, 3), PEO4 (second recurrence, 10 months post first recurrence; Patient ID, 3), PEO23 (recurrence, 7 months post chemotherapy; Patient ID, 2), and PEO14 (presentation, prior to chemotherapy; Patient ID, 2) (11), were grown in vitro. Original tissue materials were collected in a study which was approved by the Cambridge Local Research Ethics Committee (LREC), all patients gave written informed consent prior to participation (12). and mRNA of the cells was extracted and then sequenced on Illumina GAII genome analyzer (Illumina Inc., San Diego, CA, USA). Each sample was subject to three experimental repeats for statistical calculation. The raw sequence data were downloaded from the European Molecular Biology Laboratory, European Bioinformatics Institute database (http://www.ebi. ac.uk/arrayexpress/experiments/E-MTAB-691/). High-quality data were obtained by filtering raw reads with the fastx-toolkit (http://hannonlab.cshl.edu/fastx_toolkit/) (13) (Table I). The quality of the data was controlled using FastQC (http://www. bioinformatics.babraham.ac.uk/projects/fastqc/) (14). 
Table I. Data description.

\begin{tabular}{|c|c|c|c|c|c|}
\hline Sample & Read length (bp) & Patient ID & Stage & Raw bases & Bases after filtering \\
\hline PEO1 & $42 * 2$ & 2 & First recurrence & $1.3 \mathrm{G}$ & $1.2 \mathrm{G}$ \\
\hline PEO1 & $42 * 2$ & 2 & First recurrence & $1.4 \mathrm{G}$ & $1.2 \mathrm{G}$ \\
\hline PEO1 & $42 * 2$ & 2 & First recurrence & $1.7 \mathrm{G}$ & $1.5 \mathrm{G}$ \\
\hline PEO4 & $42 * 2$ & 2 & Second recurrence & $1.6 \mathrm{G}$ & $1.3 \mathrm{G}$ \\
\hline PEO4 & $42 * 2$ & 2 & Second recurrence & $1.5 \mathrm{G}$ & $1.3 \mathrm{G}$ \\
\hline PEO4 & $42 * 2$ & 2 & Second recurrence & $1.4 \mathrm{G}$ & $1.3 \mathrm{G}$ \\
\hline PEO14 & $43 * 2$ & 3 & Presentation & $1.2 \mathrm{G}$ & $1.1 \mathrm{G}$ \\
\hline PEO14 & $43 * 2$ & 3 & Presentation & $933.6 \mathrm{M}$ & $860 \mathrm{M}$ \\
\hline PEO14 & $43 * 2$ & 3 & Presentation & $1.3 \mathrm{G}$ & $1.2 \mathrm{G}$ \\
\hline $\mathrm{PEO} 23$ & $42 * 2$ & 3 & Recurrence & $1.8 \mathrm{G}$ & $1.6 \mathrm{G}$ \\
\hline PEO23 & $42 * 2$ & 3 & Recurrence & $1.7 \mathrm{G}$ & $1.5 \mathrm{G}$ \\
\hline PEO23 & $43 * 2$ & 3 & Recurrence & $701.8 \mathrm{M}$ & $670 \mathrm{M}$ \\
\hline
\end{tabular}

Marker selection. All the markers of ovarian carcinoma were selected from the Nucleotide database in National Center for Biotechnology Information (http://www.ncbi.nlm.nih.gov/). Markers were selected according to the following criteria: The nucleic acid sequence existed, the sequence type was RNA and there were supporting documents (listed in the Nucleotide database with their corresponding marker). Normal human genes were downloaded from University of California Santa Cruz (UCSC) Genome Bioinformatics (http:/genome. ucsc.edu/, version hg19). Gene family information was downloaded from the HUGO Gene Nomenclature database (http://www.genenames.org/).

Interaction network of markers pathways. Pathways enrichment analysis for all the selected markers was performed by the Kyoto Encyclopedia of Genes and Genomes (KEGG) database (15), then the enrichment pathways were linked together to form an interaction network.

Marker expression evaluation and DEMs detection. Bioinformatics protocols were applied to analyze the sequencing data. Firstly, sequencing data were mapped to marker sequences using bowtie (16) with a maximum of two mismatches in the read, at most three mismatches in a whole read, and only the best alignment reported of each read, while the rest parameters were left as default. Secondly, expression levels of markers were measured in reads per kilobase of transcript per million mapped reads (RPKM) (17). Only markers with an average RPKM value of three experimental duplicates $\geq 1$ were defined as expressed markers. Thirdly, the DEMs of the four samples were detected referring to the method developed by Audic and Claverie (18). The P-values were adjusted using the Benjamin and Hochberg correction (19) in the R programming language. According to the criteria, expressed markers with fold change $\geq 1.5$ and false discovery rate-adjusted P-value $\leq 0.01$ were defined as differentially-expressed. Spearman's coefficient was calculated to demonstrate the correlation between experimental duplicates (20).

\section{Results}

Expressed marker analysis. In the present study, a total of 563 markers were selected, all of which were documented to be associated with ovarian cancer. Therefore, it was expected that they would be expressed in the samples. The expression levels of the markers were validated by RPKM, which is a quantitative value with widespread use in RNA-seq analysis (21). As a result, 484 markers ( $85.97 \%)$ were detected to be expressed in one or more samples. It was found that the RPKM values of the markers were similar in the four samples (Fig. 1A). The majority of the markers were expressed in all four samples (411 of $484 ; \sim 84.92 \%$ ), with each sample also exhibiting specifically-expressed genes (Fig. 1B).

Certain genes in the same gene family have similar roles in metabolic processes, therefore it was inferred that they may be expressed synchronously in the process of transcription. To verify this hypothesis, all the known gene families found in UCSC were selected, and the 484 markers were categorized into 94 gene families. By comparing the RPKM values of these markers using cluster analysis (Fig. 2), it was identified that the three experimental replicates of each cell line can be clustered together. Samples of the two patients could be distinguished. However, samples of PEO14 and PEO23 could not be separated by the cluster analysis. This inferred that the differences between the two individuals had a greater effect on the expression of the marker than the differences between the tumor stages.

Pathway interaction network. A KEGG pathway interaction network was developed (Fig. 3). In the 484 expressed markers, 257 markers were detected to be enriched in 44 pathways, and 72 markers appeared in pathways associated with cancer (Fig. 3, pink). AKT2 (22,23), a putative oncogene, was enriched in the majority of the cancer-associated pathways in the network, including small cell lung cancer, non-small cell lung cancer, thyroid cancer and bladder cancer. Similarly, $E 2 F 2$ and BRCA2 also appeared in several cancer-associated pathways. With the exception of cancer, pathways associated 

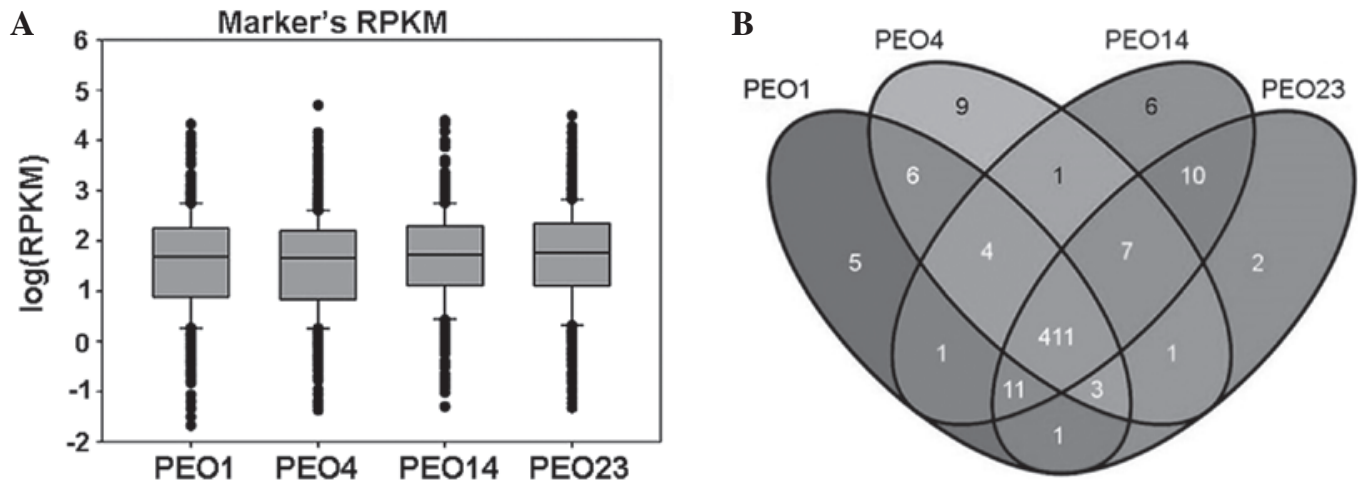

Figure 1. Statistical descriptions of expressed markers. (A) RPKM statistical descriptions of the four samples (PEO1,PEO4, PEO14 and PEO23). (B) Distribution of the markers in the four samples. Digits are the numbers of markers. RPKM, reads per kilobase of transcript per million mapped reads.

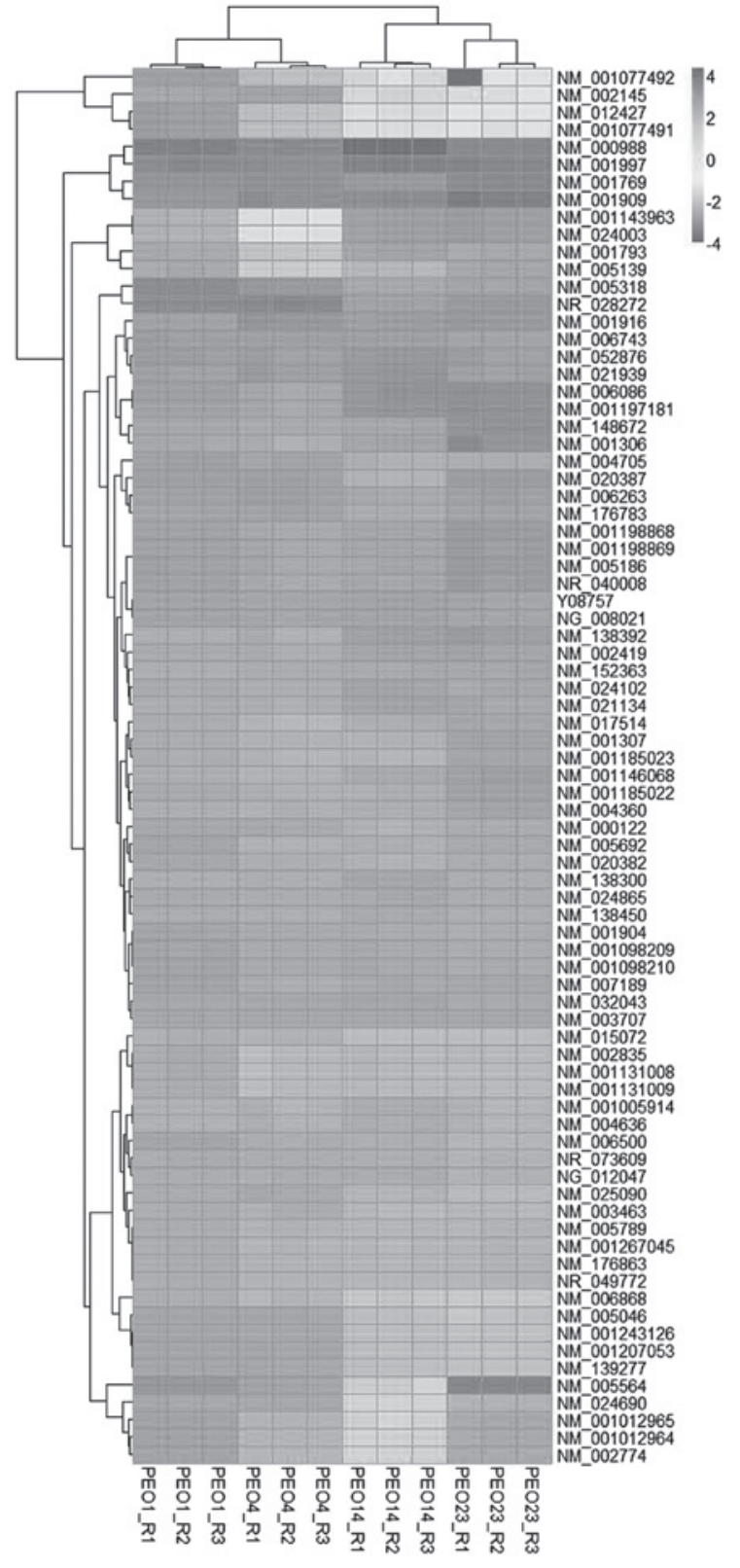

Figure 2. Heatmap of gene families of expressed markers in the 12 samples. Each cell stands for log value of RPKM. Accession numbers are indicated on the right, on the bottom are sample names, R1, R2, R3 are the three experimental duplicates and the dendrograms on the top and left demonstrate the association of samples and markers, respectively. RPKM, reads per kilobase of transcript per million mapped reads. with infection also appeared in the network with 35 markers. In the network, the p53 signaling pathway was linked with oocyte meiosis and progesterone-mediated oocyte maturation. The mTOR signaling pathway was also found to be associated with the progesterone-mediated oocyte maturation.

DEMs analysis. By comparing the changes of expression levels of markers from the presentation stage to the recurrence stage, it was found that certain markers had roles in recurrence in ovarian cancer. In the present study, the expression levels of markers between different patients with different tumor stages were compared. More DEMs were detected between two different tumor stages of the same patient, compared with between patients (Fig. 4). DEMs were detected in four pairs of comparisons, PEO14 vs. PEO23, PEO14 vs. PEO1, PEO14 vs. PEO4, and PEO4 vs. PEO1. As a result, 85 markers were found to be differentially-expressed.

\section{Discussion}

Markers associated with ovarian cancer were analyzed in the present study. To verify which markers were expressed in tumor cells, RNA-seq data analysis was applied. Of a total of 563 markers that were selected, 484 markers ( 85.97\%) were expressed in human ovarian tumor cells, which gave clear evidence that these are markers of ovarian cancer from the respect of the whole transcriptome. Differences between individuals are often omitted, but the present result demonstrated that expression levels of markers were more likely to be different between individuals than between tumors of different stages. In addition, more DEMs were detected between two different tumor stages of the same patient compared with between patients. Thus, in a future study, more samples from various ovarian cancer patients should be sequenced to prevent interindividual differences affecting the data.

In the expressed markers, the majority of the markers were enriched in pathways associated with cancer (72 markers), signaling (129 markers) and infection (35 markers). AKT2, a putative oncogene (24), was enriched in the majority of the cancer-associated pathways in the network and was first found to be aberrantly expressed in human ovarian cancer (25). Similarly, E2F2 and BRCA2 were also enriched in several cancer-associated pathways. The existence of pathways associated with infection in the pathway network once again 


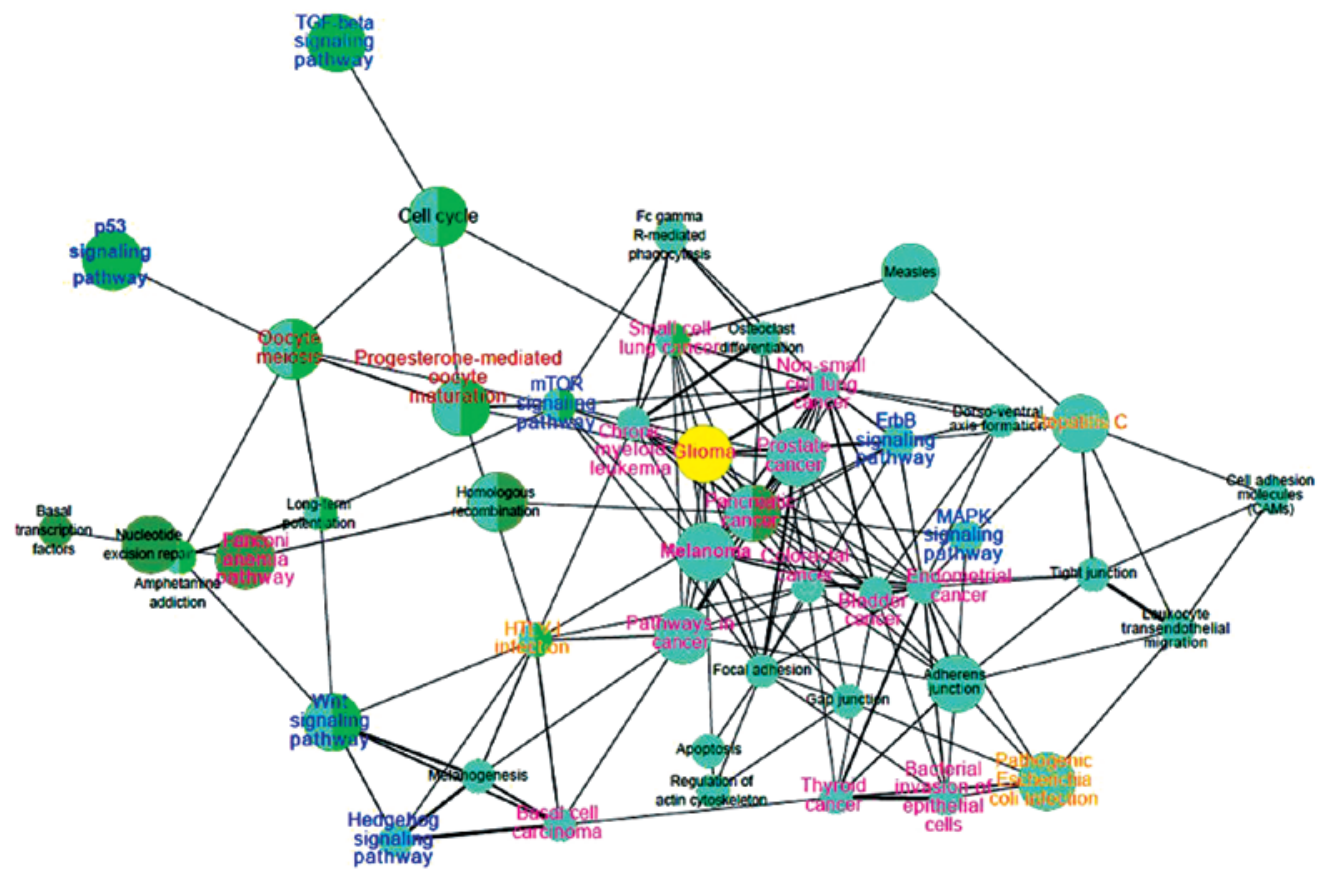

Figure 3. Pathways interaction network of expressed markers. Circle nodes stand for the pathways; lines between them show their associations. Pink words are pathways associated with cancer, blue indicates signaling pathways, yellow indicates pathways associated with infection, and the two red pathways are involved with oocytes.
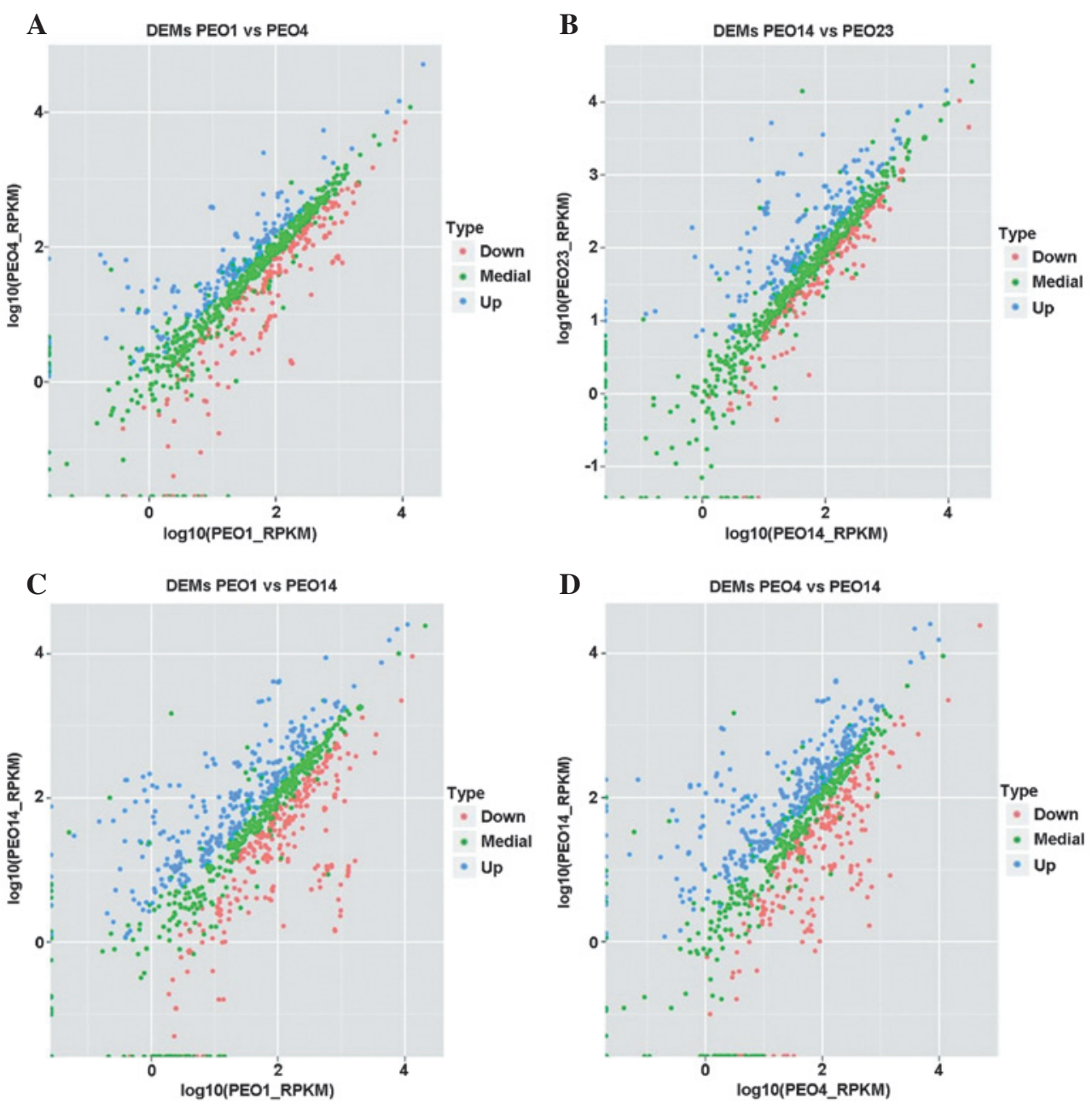

Figure 4. DEMs of four groups of comparatives. Blue points indicate markers upregulated in samples shown on the X-axis, while the red points indicate the downregulated genes and the green points indicate those without significant changes. (A) DEMs between PEO1 and PEO4 samples; (B) DEMs between PEO14 and PEO23 samples; (C) DEMs between PEO1 and PEO14 samples; (D) DEMs between PEO4 and PEO14 samples. DEMs, differentially-expressed markers. 
supported the view that external infections, such as bacteria and viruses may be associated with oncogenes in the induction of the occurrence of carcinomas (26-28). Signaling pathways also have important roles in cancer progression (29), and there were 129 detected markers in this type of pathway. Inactivation of p53 is implicated in tumor progression and numerous activated oncogenes included in the p53 signaling pathway have been elaborately investigated $(30,31)$. In the network determined in teh present study, the p53 signaling pathway was linked with oocyte meiosis and progesterone-mediated oocyte maturation (http://www.genome.jp/kegg/pathway/hsa/hsa04115.html). As has been established, the oocyte is the area where ovarian carcinoma formation is initiated, and unbalanced estrogen metabolism is hypothesized to be one of the causes of ovarian carcinoma (32). Thus, markers enriched in this pathway may have a role in the oncogenesis of ovarian cancer. The mTOR signaling pathway was also found to be associated with progesterone-mediated oocyte maturation. The deregulation of mTOR, a downstream effector of the AKT pathway, has been reported to have effects on tumor progression (33).

All the markers that were selected are associated with the presentation, development and diagnosis of ovarian cancer, according to the findings from associated studies. In total, 85 markers were abnormally expressed in tumor cells from patients who had recurrences. Of the 33 upregulated markers, TMP (34), MAL2 (35), ERCC (36), CD (37), KLK (38) and $S C A R A 3$ (39) were also observed to be over-expressed in previous studies. The $C D 9$ gene encodes a member of transmembrane 4 superfamily-tetraspanin family (40), which regulates cell surface glycoprotein function in differentiation and signal transduction. Notably, gene expression is involved in the suppression of cancer cell motility and metastasis (41). Although a study found that downregulation of $C D 9$ may be associated with the process of ovarian tumor dissemination (42), the present study demonstrated that $C D 9$ had a high expression level (RPKM) in all four samples (1290.7 in PEO14, 2141.98 in PEO1, 2283.54 in PEO4 and 4084.01 in PEO23 with a significant increase at the recurrence stage). AKTIP may have a role in apoptosis; however, at present no studies have ruled out its association with the risk of developing ovarian cancer (43). In the present study, AKTIP was overexpressed in the PEO23 cell line, with an $\sim 2$ fold increase at the recurrence stage compared with that of the PEO14 cell line. The Hox genes are a large gene family, including numerous genes located on different chromosomes in the human genome (44). In the present study, four Hox genes were found to be expressed in ovarian cancer cells, HOXA4, HOXA5, HOXD1 and HOXD3, all of which were downregulated in the recurrence stage compared with the presentation stage. In a further study, the HOXA4 gene is over-expressed in human ovarian cancer when compared with benign tumors (45). The inappropriate expression of the HOXA5 gene disrupts normal growth and differentiation programs (46). In the present study, HOXA5 was expressed in all four ovarian cancer samples with a downregulated expression level at the recurrence stage. However, there have been no previous studies, to the best of our knowledge, regarding the contribution of $H O D X 1$ and $H O D X 3$ genes to ovarian cancer, while $H O D X 9$ and $H O D X 11$ genes were reported to be significantly increased in ovarian tumor cells (47). Although all the marker genes that were selected can be connected to the progression, invasion or high risk of ovarian carcinoma, there have been no previous studies regarding genetic associations with its recurrence. The DEMs that were identified may be connected with the recurrence of human ovarian cancer by regulating the expression levels of the genes, but the exact regulatory mechanism requires further investigation.

In conclusion, DEMs of ovarian cancer samples at different stages and the associations between them can be clearly investigated by examining the RNA-seq data. These markers may provide novel prospects for further studies on ovarian cancer.

\section{References}

1. Moss EL, Hollingworth J and Reynolds TM: The role of CA125 in clinical practice. J Clin Pathol 58: 308-312, 2005.

2. Lakhani SR, Manek S, Penault-Llorca F, et al: Pathology of ovarian cancers in BRCA1 and BRCA2 carriers. Clin Cancer Res 10: 2473-2481, 2004.

3. Rafnar T, Gudbjartsson DF, Sulem P, et al: Mutations in BRIP1 confer high risk of ovarian cancer. Nat Genet 43: 1104-1107, 2011.

4. Ducros E, Mirshahi S, Azzazene D, et al: Endothelial protein C receptor expressed by ovarian cancer cells as a possible biomarker of cancer onset. Int J Oncol 41: 433-440, 2012.

5. Fung FK, Chan DW, Liu VW, Leung TH, Cheung AN and Ngan HY: Increased expression of PITX2 transcription factor contributes to ovarian cancer progression. PLoS One 7: e37076, 2012

6. Ishibashi M, Nakayama K, Yeasmin S, et al: A BTB/POZ gene, NAC-1, a tumor recurrence-associated gene, as a potential target for taxol resistance in ovarian cancer. Clin Cancer Res 14: 3149-3155, 2008

7. Rajkumar T, Soumittra N, Nancy NK, Swaminathan R, Sridevi V and Shanta V: BRCA1, BRCA2 and CHEK2 (1100 del C) germline mutations in hereditary breast and ovarian cancer families in south india. Asian Pac J Cancer Prev 4: 203-208, 2003.

8. Ghadersohi A, Odunsi K, Zhang S, et al: Prostate-derived Ets transcription factor as a favorable prognostic marker in ovarian cancer patients. Int J Cancer 123: 1376-1384, 2008.

9. Tassi RA, Calza S, Ravaggi A, et al: Mammaglobin B is an independent prognostic marker in epithelial ovarian cancer and its expression is associated with reduced risk of disease recurrence. BMC Cancer 9: 253, 2009.

10. Rahman M, Nakayama K, Rahman M, et al: Fatty acid synthase expression associated with NAC1 is a potential therapeutic target in ovarian clear cell carcinomas. Brit J Cancer 107: 300-307, 2012.

11. Ng CK, Cooke SL, Howe K, et al: The role of tandem duplicator phenotype in tumour evolution in high-grade serous ovarian cancer. J Pathol 226: 703-712, 2012

12. Ahmed AA, Mills AD, Ibrahim AE, et al: The extracellular matrix protein TGFBI induces microtubule stabilization and sensitizes ovarian cancers to paclitaxel. Cancer Cell 12: 514-527, 2007.

13. Krueger F, Kreck B, Franke A and Andrews SR: DNA methylome analysis using short bisulfite sequencing data. Nat Methods 9: 145-151, 2012.

14. Madougou S, Santcroos M, Benabdelkader A, et al: Provenance for distributed biomedical workflow execution. Stud Health Technol Inform 175: 91-100, 2012

15. Mao X, Cai T, Olyarchuk JG and Wei L: Automated genome annotation and pathway identification using the KEGG orthology (KO) as a controlled vocabulary. Bioinformatics 21: 3787-3793, 2005.

16. Langmead B, Trapnell C, Pop M and Salzberg SL: Ultrafast and memory-efficient alignment of short DNA sequences to the human genome. Genome Biol 10: R25, 2009.

17. Mortazavi A, Williams BA, Mccue K, Schaeffer L and Wold B: Mapping and quantifying mammalian transcriptomes by RNA-seq. Nat Methods 5: 621-628, 2008.

18. Audic S and Claverie JM: The significance of digital gene expression profiles. Genome Res 7: 986-995, 1997.

19. Benjamini Y and Yekutieli D: The control of the false discovery rate in multiple testing under dependency. Ann Stat 29: 1165-1188, 2001. 
20. Harr B and Schlötterer C: Comparison of algorithms for the analysis of affymetrix microarray data as evaluated by co-expression of genes in known operons. Nucleic Acids Res 34: e8, 2006.

21. Adamidi C, Wang Y, Gruen D, et al: De novo assembly and validation of planaria transcriptome by massive parallel sequencing and shotgun proteomics. Genome Res 21: 1193-1200, 2011.

22. Virtakoivu R, Pellinen T, Rantala JK, Perälä M and Ivaska J: Distinct roles of AKT isoforms in regulating $\beta 1$-integrin activity, migration and invasion in prostate cancer. Mol Biol Cell 23 . 3357-3369, 2012.

23. Dobashi Y, Kimura M, Matsubara H, Endo S, Inazawa J and Ooi A: Molecular alterations in AKT and its protein activation in human lung carcinomas. Hum Pathol 43: 2229-2240, 2012.

24. Bellacosa A, De Feo D, Godwin AK, et al: Molecular alterations of the AKT2 oncogene in ovarian and breast carcinomas. Int J Cancer 64: 280-285, 1995.

25. Altomare DA, Wang HQ, Skele KL, et al: AKT and mTOR phosphorylation is frequently detected in ovarian cancer and can be targeted to disrupt ovarian tumor cell growth. Oncogene 23 : 5853-5857, 2004.

26. Chu CM: Natural history of chronic hepatitis B virus infection in adults with emphasis on the occurrence of cirrhosis and hepatocellular carcinoma. J Gastroen Hepatol 15: E25-E30, 2000.

27. Parsonnet J, Friedman GD, Vandersteen DP, et al: Helicobacter pylori infection and the risk of gastric carcinoma. N Engl J Med 325: 1127-1131, 1991.

28. Sobhani I, Walker F, Roudot-Thoraval F, et al: Anal carcinoma: incidence and effect of cumulative infections. Aids 18: 1561-1569, 2004

29. Dreesen O and Brivanlou AH: Signaling pathways in cancer and embryonic stem cells. Stem Cell Rev 3: 7-17, 2007.

30. Osman I, Drobnjak M, Fazzari M, et al: Inactivation of the p53 pathway in prostate cancer: impact on tumor progression. Clin Cancer Res 5: 2082-2088, 1999.

31. Puzio-Kuter AM, Castillo-Martin M, Kinkade CW, et al: Inactivation of $\mathrm{p} 53$ and Pten promotes invasive bladder cancer. Genes Dev 23: 675-680, 2009.

32. Ho SM: Estrogen, progesterone and epithelial ovarian cancer. Reprod Biol Endocrinol 1: 73, 2003.

33. Pópulo H, Lopes JM and Soares P: The mTOR signalling pathway in human cancer. Int J Mol Sci 13: 1886-1918, 2012.

34. Guerrero K, Wang Z, Bachvarova M, et al: A novel genome-based approach correlates TMPRSS3 overexpression in ovarian cancer with DNA hypomethylation. Gynecol Oncol 125: 720-726, 2012.
35. Byrne J, Maleki S, Hardy JR, et al: MAL2 and tumor protein D52 (TPD52) are frequently overexpressed in ovarian carcinoma, but differentially associated with histological subtype and patient outcome. BMC cancer 10: 497, 2010.

36. Guo Y, Fu P, Zhu H, et al: Correlations among ERCC1, XPB, UBE2I, EGF, TAL2 and ILF3 revealed by gene signatures of histological subtypes of patients with epithelial ovarian cancer. Oncol Rep 27: 286-292, 2012.

37. Hwang JR, Jo K, Lee Y, Sung BJ, Park YW and Lee JH: Upregulation of CD9 in ovarian cancer is related to the induction of TNF- $\alpha$ gene expression and constitutive NF- $\kappa \mathrm{B}$ activation. Carcinogenesis 33: 77-83, 2012.

38. Seiz L, Dorn J,Kotzsch M, et al: Stromal cell-associated expression of kallikrein-related peptidase 6 (KLK6) indicates poor prognosis of ovarian cancer patients. Biol Chem 393: 391-401, 2012

39. Bock AJ, Nymoen DA, Brenne K, Kærn J and Davidson B: SCARA3 mRNA is overexpressed in ovarian carcinoma compared with breast carcinoma effusions. Hum Pathol 43: 669-674, 2012.

40. Chuan Y, Pang ST, Bergh A, Norstedt G and Pousette A: Androgens induce CD-9 in human prostate tissue. Int $\mathrm{J}$ Androl 28: 291-296, 2005.

41. Cook GA, Wilkinson DA, Crossno JT Jr, Raghow R and Jennings LK: The tetraspanin CD9 influences the adhesion, spreading and pericellular fibronectin matrix assembly of chinese hamster ovary cells on human plasma fibronectin. Exp Cell Res 251: 356-371, 1999.

42. Furuya M, Kato H, Nishimura N, et al: Down-regulation of CD9 in human ovarian carcinoma cell might contribute to peritoneal dissemination: morphologic alteration and reduced expression of $\beta 1$ integrin subsets. Cancer Res 65: 2617-2625, 2005.

43. Notaridou M, Quaye L, Dafou D, et al: Common alleles in candidate susceptibility genes associated with risk and development of epithelial ovarian cancer. Int J Cancer 128: 2063-2074, 2011.

44. Acampora D, D'esposito M, Faiella A, et al: The human hox gene family. Nucleic Acids Res 17: 10385-10402, 1989.

45. Ota T, Klausen C, Salamanca MC, Woo HL, Leung PC and Auersperg N: Expression and function of HOXA genes in normal and neoplastic ovarian epithelial cells. Differentiation 77: 162-171, 2009.

46. Gendronneau G, Boucherat O, Aubin J, Lemieux M and Jeannotte L: The loss of Hoxa5 function causes estrous acyclicity and ovarian epithelial inclusion cysts. Endocrinology 153: 1484-1497, 2012

47. Morgan R, Plowright L, Harrington KJ, Michael A and Pandha HS: Targeting HOX and PBX transcription factors in ovarian cancer. BMC cancer 10: 89, 2010. 